UDC 532.536

\title{
LINEAR AND NONLINEAR INSTABILITY OF FLOW IN CHANNEL OCCUPIED POROUS MEDIA
}

\author{
Avramenko A.A., corresponding member of NASU, Dmitrenko N.P., Kovetskaya Y.Y. \\ Institute of Engineering Thermophysics of the National Academy of Sciences of Ukraine, \\ st. Zhelyabova, 2a, Kyiv, 03680 Ukraine
}

В статті досліджена лінійна та нелінійна гідродинамічна нестійкість потоку в каналі, заповненому пористим середовищем. Ефекти лінійної нестійкості розглянуті, використовуючи метод лінійних збурень. Нелінійна нестійкість потоку розглянута, використовуючи ренормалізірований вираз для коефіцієнта кінематичної в'язкості.

References 21, figures 2.
В статье исследована линейная и нелинейная гидродинамическая неустойчивость потока в канале, заполненном пористой средой. Эффекты линейной неустойчивости рассмотрены, используя метод линейных возмущений. Нелинейная неустойчивость потока рассмотрена, используя ренормализированное выражение для коэффициента кинематической вязкости.
The paper investigates linear and nonlinear hydrodynamic instability of flow in channel ocuped porous medium. The effects of linear instability are considered using the method of linear perturbations. The nonlinear instability of the flow is considered using the renormalized expression for the coefficient of the kinematic viscosity.

Key words: renormalized analysis, mathematical model, porosity, instability, turbulence.

$c_{F}$ - Forchheimer's coefficient;

$d$ - dimensionality of space;

D - constant;

$E$ - spectrum of turbulent energy;

$f$ - solenoidal force;

$F$ - Fourier transform of solenoidal force;

$K$ - permeability;

$p$-pressure;

$P$ - pressure transform;

$s$ - direction cosines of velocity vector;

$S-$ Fourier transform of the direction cosines of velocity

vector;

$t$ - time;

$u, v, w$ - velocity component;

$x, y, z$ - cartesian coordinates;

$U-$ Fourier transform of velocity;

$V$ - velocity vector;

$\beta, \kappa$ - wave numbers;

$\varepsilon$-dissipation rate;

$\mu$ - effective coefficient of dynamic viscosity;

\section{Introduction}

The investigation into the physics of flow in a porous medium has provided the basis for many theoretical and practical studies in various fields such as the mechanics of grounds, hydrology of ground water, oil engineering, industrial filtration, powder metallurgy, atomic power engineering, etc. For some time now the processes of turbulent flow in a porous medium have been studied closely and intensively. In $[1,2]$, the derivation of the $k-\varepsilon$ model equations is set out for modeling the processes of macroscopic turbulence in porous media. The results of numerical simulation obtained with the use of the model $v$ - effective coefficient kinematic viscosity ;

$\rho$ - density;

$\tau$ - positive parameter;

$\varphi$ - porosity;

$\omega-$ frequency.

Indices:

$c, h, k, l, m, n, r, s, o-$ projections on coordinates;

$e$ - effective parameter;

$t$ - turbulent parameter;

0 - start point.

Complexes:

$D a=\frac{K}{h^{2}}-$ the Darcy criterion;

$M=(\mathrm{Da})^{-1 / 2}$;

$\operatorname{Re}=\frac{u_{\infty} h}{v}-$ Reynolds criterion;

$\Lambda=\varphi^{3 / 2} c_{F} \frac{u_{\infty} h}{v_{f} \sqrt{J}}$.

proposed in [1,2] are presented in [3]. In [4-7], various aspects of macroscopic modeling of turbulence in homogeneous porous media are considered. Using the procedure of averaging over the time, the authors of [4] obtained an equation for the kinetic energy of turbulence. Experimental investigation of the chaotic behavior of flow through porous media in time is described in [8]. In that investigation, a porous medium was considered as a pencil of narrow tubes. In [9], Barr proposed a technique for determining the origination of turbulence in porous media and calculating the effective permeability. A one-equation model for calculating two-dimensional turbulent flow through porous media was suggested in [10]. The model is based on the 
assumption that the turbulent energy generation term in the turbulent equation for kinetic energy is proportional to the cubed velocity. Semiempirical modeling of flow and of heat transfer in porous media is considered in [11]. Calculations at a low level of turbulence that may occur in a porous layer heated from below are presented in [12]. Work [13] contains the results of direct numerical simulation of turbulent flow in a pipe with porous and liquid media. In [14] the authors investigated the influence of turbulence effects on flow through a pseudoporous medium by numerically solving the Reynolds-averaged Navier-Stokes equations with the use of the $k-\varepsilon$ model of turbulence. The two-dimensional porous medium represented a random arrangement of solid particles.

As it is known, flow in a porous medium can have a laminar, transient, and turbulent character depending on the Reynolds number and the porosity parameters of the medium. For adequately describes the above-mentioned regimes it is necessary to know the conditions under which flow in a porous medium loses its stability. In [15], the linear instability of a laminar flow in a plane channel filled with a porous medium was studied. The dependence of the critical
Reynolds number on the medium porosity and permeability was analyzed numerically. The hydrodynamic instability of flow in a hyperporous medium was considered in [16]. The dependence of the critical Reynolds number on the medium porosity and Knudsen number was analyzed.

The purpose of this paper is determinating the parameters of the linear and nonlinear hydrodynamic instability of flow in occupied porous medium channel (fig. 1.) that allow to optimize the choice of various geometric parameters of the media for their realization in technological processes.

The theory of renormalized groups (RNG), used in the present article, has been developed in quantum physics. This theory was applied to studying turbulent flows in [18]. Using the RNG theory, Yakhot and Orzag [19] developed a closed model of turbulence.

The renormalization group method was also applied to studying turbulent flows in a porous medium in [20]. In the case of a porous medium, this approach made it possible to reveal the physical essence of the effect exerted by the Forchheimer component on the behavior of flow turbulence not resorting to empirical information.

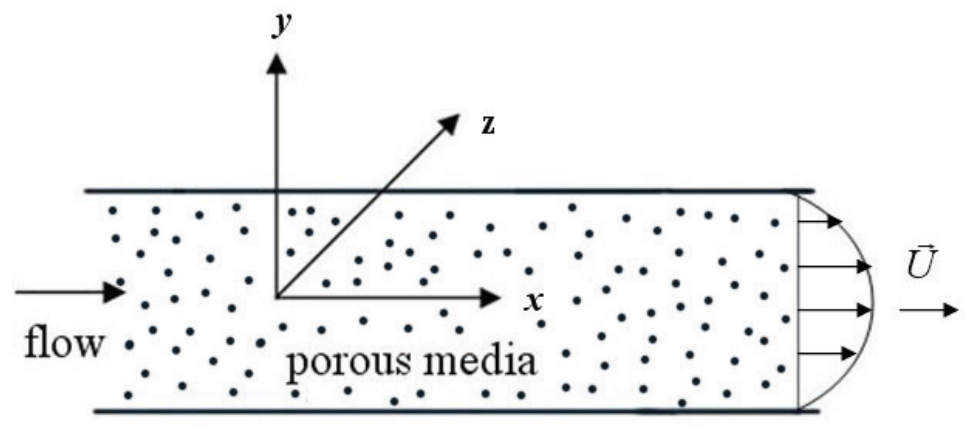

Fig. 1. Calculating domain scheme.

\section{Linaer instability}

To describe the porous medium, one can use the DarcyBrickman-Forchaymer model [17]. This model was used as the basis for studying the hydrodynamic instability in a macroporous medium using a two-dimensional approach [15]. The present study uses a three-dimensional approximation.

The flow dynamics in a porous medium is described by a system of modified Navier-Stokes differential equations and the continuity equation.

$$
\begin{aligned}
& \frac{\partial u}{\partial t}+u \frac{\partial u}{\partial x}+v \frac{\partial u}{\partial y}+w \frac{\partial u}{\partial z}=-\frac{1}{\rho} \frac{\partial p}{\partial x}+v_{t} \nabla^{2} u+v_{f} \frac{\varphi}{K} u-\varphi^{2} \frac{c_{F}}{\sqrt{K}}|V| u,(1, \mathrm{a}) \\
& \frac{\partial v}{\partial t}+u \frac{\partial v}{\partial x}+v \frac{\partial v}{\partial y}+w \frac{\partial v}{\partial z}=-\frac{1}{\rho} \frac{\partial p}{\partial y}+v_{t} \nabla^{2} v+v_{f} \frac{\varphi}{K} v-\varphi^{2} \frac{c_{F}}{\sqrt{K}}|V| v,(1, \mathrm{~B}) \\
& \frac{\partial w}{\partial t}+u \frac{\partial w}{\partial x}+v \frac{\partial w}{\partial y}+w \frac{\partial w}{\partial z}=-\frac{1}{\rho} \frac{\partial p}{\partial y}+v_{t} \nabla^{2} w+v_{f} \frac{\varphi}{K} w-\varphi^{2} \frac{c_{F}}{\sqrt{K}}|V| w,(1, \mathrm{c}) \\
& \frac{\partial u}{\partial x}+\frac{\partial v}{\partial y}+\frac{\partial w}{\partial z}=0 .
\end{aligned}
$$

This system of equations includes terms that take into account the hydraulic resistance caused by the porosity of the medium. The first one describes the Darcy line resistance and the second describes nonlinear resistance of Forchimer.

To determine the criteria of hydrodynamic instability one can use the method of linear perturbations. According to this method, the flow parameters are represented in the following form:

$$
\begin{aligned}
& u=\hat{U}(y)+u^{\prime}(t, x, y, z), v=v^{\prime}(t, x, y, z), \\
& w=w^{\prime}(t, x, y, z), p=\hat{P}(x)+p^{\prime}(t, x, y, z),
\end{aligned}
$$

where $\hat{U}, \hat{P}$ are the parameters of the basic unperturbed flow. $u^{\prime}, v^{\prime}, w^{\prime}, p^{\prime}-$ perturbation parameters. The basic unperturbed flow is determined by the quantities: $u=\hat{U}(y), v=w=0, p=\hat{P}(x)$.

We substitute expressions (2) in the system of basic differential equations (1), discard the quadratic terms relative to the components of the velocity of the disturbing motion. The perturbed quantities $u^{\prime}, v^{\prime}, w^{\prime}, p^{\prime}$ can be represented as $3 \mathrm{D}$ waves in the form of such functions: 
$u^{\prime}=u_{1}(y) \exp (i(\alpha x+\gamma z-\beta t))$,

$v^{\prime}=v_{1}(y) \exp (i(\alpha x+\gamma z-\beta t))$,

$w^{\prime}=w_{1}(y) \exp (i(\alpha x+\gamma z-\beta t))$.

$p^{\prime}=p_{1}(y) \exp (i(\alpha x+\gamma z-\beta t))$.

where $\beta$ - is a complex quantity and can be represented in such a form $\beta=\beta_{r}+i \beta_{i}$, where $\beta$ is the circular frequency of the individual oscillation, $\beta_{i}$ is the growth coefficient, $\alpha$ and $\gamma$ are the wave numbers, $u_{1}, v_{1}, w_{1}, p_{1}$ are the amplitudes. In the course of mathematical transformations, amplitudes $u_{1}$, $w_{1}, p_{1}$ were eliminated, and an equation of the fourth order was obtained:

$$
\begin{aligned}
& -\frac{\Lambda M \widetilde{\mho}_{1}^{\prime}}{\widetilde{\alpha}}+\widetilde{\mathrm{v}}_{1}^{\prime}\left(-i \widetilde{c} \widetilde{k}^{2}+\frac{\widetilde{k}^{4}}{\operatorname{Re} \widetilde{\alpha}}+\frac{\widetilde{k}^{2} M^{2}}{\operatorname{Re} \widetilde{\alpha}} i \widetilde{k}^{2} \widetilde{U}+\frac{\Lambda M}{\widetilde{\alpha}} i \widetilde{U}^{\prime \prime}\right)+ \\
& \widetilde{v}^{\prime \prime}\left(i \widetilde{\widetilde{c}}-\frac{2 \widetilde{k}^{2}}{\operatorname{Re} \widetilde{\alpha}}-\frac{M^{2}}{\operatorname{Re} \widetilde{\alpha}}-i \widetilde{U}-\frac{\Lambda M}{\widetilde{\alpha}}\right)+\frac{\widetilde{\sigma}^{\prime \prime \prime \prime}}{\operatorname{Re} \widetilde{\alpha}}=0,
\end{aligned}
$$

where the prime denotes differentiation with respect to $\tilde{y}$ $(\widetilde{y}=y / h)$. In equation (4) $k^{2}=\alpha^{2}+k^{2}, h$ is channel half widht, $u$-velocity in the center of the channel $\tilde{\alpha}=\alpha h$, $\widetilde{k}=k h, \widetilde{U}=\hat{U} / u_{\infty}, \widetilde{c}=\beta / \alpha u_{\infty}$.

In the case when $M=\Lambda=0$ equation (4) is transformed into a classical equation for calculating the instability of a pure liquid.

In order to determine the criterion of hydrodynamic instability, it is necessary to study equation (4) for eigenvalues. The boundary conditions have the following form:

$\widetilde{y}=-1, \widetilde{v}=v^{\prime}=0$,

$\widetilde{y}=1, \widetilde{v}=v^{\prime}=0$.

Further, critical stability parameters were calculated for different sets of values of the parameters $M$ and $\Lambda$. The results of calculations are presented in fig. 2 .

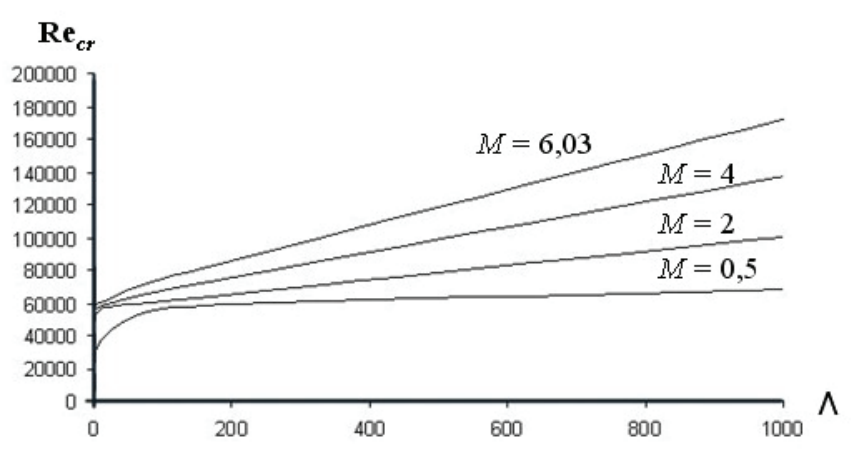

Fig. 2. Dependence of the critical Reynolds number on the parameters $M$ and $\Lambda$.

In case $M=\Lambda=0$ the critical parametr of stability is $\operatorname{Re}_{c r}=5725$, which agrees with the data of [19]. It can be seen from the figure if the parameters $M$ and $\Lambda$ increase, the value of $\mathrm{Re}_{\text {cr }}$ increases as weel. This is due to the fact that as the parameters $M$ increase and the velocity profile becomes more filled and this, in accordance with the second Rayleigh theorem on the stability of the flow, leads to the stabilization of the flow and to an increase in the value of the critical Reynolds number.

Further we consider nonlinear effects of instability which can occur after linear regime of instability. The nonlinear effects cannot be described by the Orr-Sommerfeld linear equations. To carry out an analysis of the indicated stage of instability in a porous medium, we may avail ourselves of dependence for the turbulent viscosity. In order to receive equation for turbulent viscosity we use RNG theory.

\section{Renormalized group transformation}

For it instability analysis one can use RNG approach. As is shown in [1], the equation of flow motion in a porous medium involves a term that accounts for the linear hydrodynamic resistance and is described by the Darcy law, terms that account for the Forchheimer hydrodynamic resistance, and Brinkman's correction. We rewrite equation (1) in this form $\left(\frac{\partial}{\partial t}+v_{0} \frac{\varphi}{K}-J v_{0} \nabla^{2}\right) u_{n}=f_{n}-\frac{1}{\rho} \frac{\partial p}{\partial x_{n}}+\frac{\partial u_{n} u_{m}}{\partial x_{m}}-H|\mathbf{V}| u_{n}$,

$\frac{\partial u_{m}}{\partial x_{m}}=0$,

where $H=\varphi^{2} \frac{c_{F}}{\sqrt{K}}, J=\mu_{e} / \mu$ is a viscosity ratio and $\mathbf{V}$ is the velocity vector. The projection of the velocity vector on the coordinate axis is described by the expression

$u_{n}=|\overrightarrow{\mathbf{V}}| \cos \left(\hat{\mathbf{V}} u_{n}\right)=|\mathbf{V}| s_{n}$,

where $s$ is the direction cosine.

We apply the Fourier transformation to the velocity, pressure, and force parameters in Eq. (6):

$$
\begin{aligned}
& u_{n}=\frac{1}{(2 \pi)^{d+1}} \int_{\kappa \leq \kappa_{c}} d^{d} \kappa \int d \omega \mathrm{U}_{n}(\vec{\kappa}, \omega) \exp (i \vec{\kappa} \cdot \overrightarrow{\mathrm{x}}-i \omega t), \\
& p=\frac{1}{(2 \pi)^{d+1}} \int_{\kappa \leq \kappa_{c}} d^{d} \kappa \int d \omega \mathrm{P}(\vec{\kappa}, \omega) \exp (i \vec{\kappa} \cdot \overrightarrow{\mathrm{x}}-i \omega t), \\
& u_{n} u_{m}=\frac{1}{(2 \pi)^{d+1}} \int_{\kappa \leq \kappa_{c}} d^{d} \kappa \int d \omega \mathrm{W}_{n m}(\vec{\kappa}, \omega) \exp (i \vec{\kappa} \cdot \overrightarrow{\mathrm{x}}-i \omega t),
\end{aligned}
$$


$f_{n}=\frac{1}{(2 \pi)^{d+1}} \int_{\kappa \leq \kappa_{c}} d^{d} \kappa \int d \omega F_{n}(\vec{\kappa}, \omega) \exp (i \vec{\kappa} \cdot \overrightarrow{\mathrm{x}}-i \omega t)$,

$s_{n}=\frac{1}{(2 \pi)^{d+1}} \int_{\kappa \leq \kappa_{c}} d^{d} \kappa \int d \omega S_{n}(\vec{\kappa}, \omega) \exp (i \vec{\kappa} \cdot \overrightarrow{\mathrm{x}}-i \omega t)$,

$S_{n} u_{m}^{2}=\frac{1}{(2 \pi)^{d+1}} \int_{\kappa \leq \kappa_{c}} d^{d} \kappa \int d \omega S_{s_{n} u^{2}}(\vec{\kappa}, \omega) \exp (i \overrightarrow{\mathrm{k}} \cdot \overrightarrow{\mathrm{x}}-i \omega t)$,

where $\vec{\kappa}$ - vector of wave number, $\vec{x}-$ vector of point coordinate.

Substitution eqs. (8) into Eqs. (6) gives

$G_{0}^{-1} U_{n}=F_{n}-i \kappa_{n} \frac{P}{\rho}-i \kappa_{m} W_{n m}-H W_{s_{n} u^{2}}$,

where $G_{0}=\left(-i \omega+\frac{v_{0}}{K}+J v_{0} \kappa^{2}\right)^{-1}$.

Then we apply renormalization procedure to eq. (9). The procedure of renormalization analysis consists of the subdivision of the velocity and force fields into fast and slow modes [21]. As a result, we obtain an expression for the fast and slow modes. Next, we exclude the fast modes from equation for fast modes. Using the averaging rules [21] we integrate the resalting expression. After that we have

$$
\begin{aligned}
& \Delta v(\widetilde{\kappa})=-8 \lambda_{0}^{2} \kappa^{-2} J^{-1} D_{0} M_{s m l}^{<}(\kappa) \int \frac{d \widetilde{\sigma}}{(2 \pi)^{d+1}} \cdot \\
& \cdot G_{0}(\widetilde{\sigma}-\widetilde{\kappa}) \frac{\left|G_{0}(\widetilde{\sigma})\right|^{2} M_{l t s}^{>}(\kappa-\sigma) M_{m t}(\sigma)}{\sigma^{d-4+\varepsilon^{*}}}
\end{aligned}
$$

and

$$
\begin{aligned}
& \Delta N=8 \lambda_{0}^{2} H M_{r m l}^{<}(\kappa) D_{0} \\
& \int \frac{G_{0}(\widetilde{\kappa}-\widetilde{\sigma})\left|G_{0}(\widetilde{\sigma})\right|^{2} M_{n h}^{>}(\kappa-\sigma) M_{m r}(\sigma) d \widetilde{\sigma}}{(2 \pi)^{d+1} \sigma^{d-4+\varepsilon^{*}}} \int \frac{\mathrm{S}_{h}(\widetilde{\beta}) U_{n}^{<}(\widetilde{\kappa}-\widetilde{\beta})}{(2 \pi)^{d+1}} d \widetilde{\beta} .(11)
\end{aligned}
$$

Expression (11) describes the renormalized component of the Forchheimer nonlinear resistance. The process of renormalization continues up to a fixed point. As this fixed point is approached, we get

$$
\begin{aligned}
& G^{-1}(\widetilde{\kappa}) U_{n}^{<}(\widetilde{\kappa})=F_{n}^{<}(\widetilde{\kappa})+\lambda_{0}\left[M_{n m l}^{<}(\kappa) \int \frac{d \widetilde{\sigma}}{(2 \pi)^{d+1}} U_{m}^{<}(\widetilde{\sigma}) U_{l}^{<}(\widetilde{\kappa}-\widetilde{\sigma})-\right. \\
& \left.H M_{n h}^{<}(\kappa) \int \frac{d \widetilde{\sigma} d \widetilde{\beta}}{(2 \pi)^{2 d+2}} S_{h}(\widetilde{\beta}) U_{r}^{<}(\widetilde{\sigma}) U_{r}^{<}(\widetilde{\kappa}-\widetilde{\sigma}-\widetilde{\beta})\right],
\end{aligned}
$$

where

$$
G(\widetilde{\kappa})=\left[-i\left(\omega-\frac{\Delta N}{i U_{n}^{<}(\widetilde{\kappa})}\right)+\frac{v_{0}}{K}+J \kappa^{2}\left(v_{0}+\Delta v\right)\right]^{-1} .
$$

The next step is the determination of the turbulent coefficient of kinematic viscosity with account for the porosity and for Forchheimer's correction. To obtain a differential equation that would describe the effective viscosity, we compute integral (10) over the entire spectrum of frequencies. in form:

As a result, we get the renormalized viscosity equation
$\Delta \nu=A_{d} J^{-3} \frac{\lambda_{0}^{2} D_{0}}{v_{0}^{2} \kappa_{c}^{\varepsilon^{*}}} \frac{\exp \left(\varepsilon^{*} \tau\right)-1}{\varepsilon^{*}}-$
$-B_{d} J^{-4} \frac{\lambda_{0}^{2} D_{0}}{v_{0}^{2} \kappa_{c}^{\varepsilon^{*}+2}(K / \varphi)} \frac{\exp \left(\left(2+\varepsilon^{*}\right) \tau\right)-1}{2+\varepsilon^{*}}$,

where

$$
A_{d}=\frac{S_{d}}{(2 \pi)^{d}} \frac{d-1}{2(d+2)}, \quad B_{d}=\frac{S_{d}}{(2 \pi)^{d}} \frac{d^{2}-d+3}{d(d+2)} .
$$

In order to obtain a differential equation for the effective viscosity, we differentiate (14) with respect to $\tau$ for $\tau \rightarrow 0$ :

$$
\frac{d v}{d \tau}=A_{d} J^{-3} \frac{\lambda_{0}^{2} D_{0}}{v^{2} \kappa_{c}^{\varepsilon^{*}}}\left(1-\frac{2 \varphi}{J K \kappa_{c}^{2}} \frac{d^{2}-d+3}{d(d-1)}\right) \text {. }
$$

The subscript " 0 " is omitted, since the renormalization procedure takes place when $v\left(\kappa_{c}\right) \rightarrow v\left(\kappa_{c}^{\prime}\right)$.

With account for the boundary condition $v(\infty)=0$, integration of (16) leads to the equation

$$
v=\left(\frac{3 A_{d} D_{0}}{J^{3} \kappa_{c}^{\varepsilon^{*}}}\left(\frac{1}{\varepsilon^{*}}-\frac{2 \varphi}{J K \kappa_{c}^{2}\left(2+\varepsilon^{*}\right)} \frac{d^{2}-d+3}{d(d-1)}\right)\right)^{1 / 3},
$$

which is an expression for the renormalized effective viscosity with account for the porosity of the medium.

Next, we exclude the wave number from (17). To do this, we calculate the turbulence energy spectrum from the formula of [20]. Substituting the expression for the correlation function of effective random forces and taking into account the expression for the effective viscosity with account for the porosity of the medium (17), we obtain

$$
\begin{aligned}
& E(\kappa)=\frac{d-1}{2} J\left(\frac{\varepsilon *}{3 A_{d}}\right)^{1 / 3} \frac{S_{d}}{(2 \pi)^{d}} D_{0}^{2 / 3} \kappa^{1-\frac{2 \varepsilon^{*}}{3}} . \\
& \left(1-\frac{2 \varphi \varepsilon^{*}}{J K \kappa^{2}\left(2+\varepsilon^{*}\right)} \frac{d^{2}-d+3}{d(d-1)}\right)^{-1 / 3} .
\end{aligned}
$$

Just as in the expression for turbulent viscosity, we replace $\kappa_{c}$ by $\kappa$ in eq. (18). In the case of $\varepsilon^{*}=4$ and $K \rightarrow \infty$, a porous medium is absent, and Eq. (18) is transformed into the Kolmogorov law.

Then from eq. (17) one can get

$$
v_{t}=v_{t 0}\left(1-\frac{16\left(3+(-1+d) d \varepsilon^{*} \varphi\right.}{27 C_{K}^{3} d(1+d)_{2} \mathrm{~F}_{1}^{3}\left(\frac{1}{3}, \frac{1}{3}, \frac{4}{3}, \frac{\varphi}{J K \kappa_{c}^{2}} \frac{d^{2}-d+3}{d(d-1)}\right) J^{4}\left(2+\varepsilon^{*}\right)} \frac{k^{3}}{K \varepsilon^{2}}\right)^{\frac{1}{3}} \text {, }
$$

where $v_{\text {t0 }}$ is defined in [20]. As it is seen from Eq. (19), at certain values of the parameters of flow and of the properties of a porous medium, the turbulent viscosity may degenerate. This problem will be considered below.

\section{Nonlinear isntability}

In order to carry out a non-linear analysis of the instability in porous medium we may use equation (17) for the renormalized turbulent viscosity. It follows from (17) that this viscosity is equal to zero, i.e., all nonlinear perturbations attenuate provided that 


$$
K<K_{c r}=\frac{2 \varphi \varepsilon^{*}}{J \kappa_{c}^{2}\left(2+\varepsilon^{*}\right)} \frac{d^{2}-d+3}{d(d-1)} .
$$

Eliminating the wave number from (20) and using a Gaussian filter

$$
L=\frac{2 \pi}{\kappa_{c}}
$$

we obtain the instability criterion in the form of the Darcy number:

$$
\begin{aligned}
& \mathrm{Da}<\mathrm{Da}_{c r}=\frac{\varphi \varepsilon^{*}}{2 \pi^{2} J\left(2+\varepsilon^{*}\right)} \frac{d^{2}-d+3}{d(d-1)}, \\
& \mathrm{Da}=\frac{K}{d_{s t}^{2}},
\end{aligned}
$$

where the size $d_{\text {st }}$ is the average size of the particle or the diameter of the filter that forms porous medium. This size can be used as Gaussian filter.

If for the permeability we use the Kozeny relation [17]

$$
K=\frac{d_{s t}^{2} \varphi^{3}}{180\left(1-\varphi^{2}\right)}
$$

and the expression for the viscosity ratio

$$
J=\frac{1}{\varphi} \text {, }
$$

we obtain the following equation for the critical value of the porosity:

$$
\varphi_{c r}=\frac{360+\left(\pi^{2}+120\right) d(d-1)+\pi \sqrt{d(d-1)\left(720+\left(\pi^{2}+240\right) d(d-1)\right)}}{120\left(d^{2}-d+3\right)} .
$$

For three-dimensional flow Eq. (26) yields

$$
\varphi_{c r}^{3 D}=0,72 \text {, }
$$

for two-dimensional flow we have

$\varphi_{c r}^{2 D}=0,775$.

A comparison of (27) and (28) shows that the threedimensional flow is less stable in comparison with the twodimensional one. This conclusion is directly opposite to the Squire stability theorem for the linear stage of instability development, in porous media too.

\section{Conclusions}

Using the method of the linear perturbations, equation for disturbing amplitudes of motion is obtained. This equation makes it possible to analyze the influence of the parameters $M$ and $\Lambda$ on the linear instability of the flow in a porous medium. With the help of the RNG approach, an expression for the kinematic viscosity was obtained. The resulting expression was used to determine the criterion of nonlinear instability. The results of instability analysis make possible to optimize the choice of various (geometric) characteristics of the porous medium for the realization of flows in various technological processes.

1. Antohe, B.V., Lage, J.L. A general two-equation macroscopic model for incompressible flow in porous media. // International Journal of Heat and Mass Transfer. 1997. - № 40.- P. 3013-3024.
2. Getachew D., Minkowycz W.J., Lage J.L. A modified form of the model for turbulent flows of an incompressible fluid in porous media // International Journal of Heat and Mass Transfer. - 2000. - №43. - P. 2909-2915.

3. Chung K.Y., Lee K.S., Kim W.S. Modified macroscopic turbulence modeling for the tube with channel geometry in porous media // Numerical Heat Transfer A.2003. - № 43.- P. 659-668.

4. de Lemos M.J.S., Pedras M.H.J. Recent mathematical models for turbulent flow in saturated rigid porous media. // ASME J Fluids Engineering. - 2001. Vol.123(4). - P. 935-940.

5. de Lemos M.J.S., Pedras M.H.J. On the mathematical description and simulation of turbulent flow in a porous medium formed by an array of elliptic rods. // ASME J Fluids Engineering. - 2001a. - Vol.123(4). P. 941-947.

6. de Lemos M.J.S., Pedras M.H.J. Macroscopic turbulence modeling for incompressible flow through undeformable porous media. // International Journal of Heat and Mass Transfer. - 2001b.- №. 44.- P. 1081-1093.

7. de Lemos M.J.S., Pedras M.H.J. Simulation of turbulent flow in porous media using a spatially periodic array and a low Re two-equation closure. // Numerical Heat Transfer. - 2001c. - Part A. - Vol.39. - P. 35-59.

8. Masuoka T., Takatsu Y., Inoue T. Chaotic behavior and transition to turbulence in porous media. // Microscale Thermophysical Engineering. - 2002. - Vol.6. - P. 347-357. 9. Barr D.W. Turbulent flow through porous media. // Ground Water. - 2001. - Vol.39. - P. 646-650.

10. Alvarez G., Bournet P.E., Flick D. Two-dimensional simulation of turbulent flow and heat transfer through stacked spheres. // International Journal of Heat and Mass Transfer. - 2003. - №.46. - P. 2459-2469.

11. Flick D., Leslous A., Alvarez G. Semi-empirical modeling of turbulent fluid flow and heat transfer in porous media. // International Journal of Refrigeration. - 2003. Vol.26. - P. 349-359.

12. Vadasz $P$. Small and moderate Prandtl number convection in a porous layer heated from below. // International Journal of Energy Research. - 2003. - Vol.27. P. 941-960.

13. Hahn S., Je J., Choi H. Direct numerical simulation of turbulent flow with permeable walls. // J Fluid Mech. 2002. - Vol.450. - P. 259-285.

14. Macedo H.H., Costa U.M.S. Almeida M.P. Turbulent effects on fluid flow through disordered porous media // Physica A. - 2001. - Vol.299. - P. 371-377.

15. Avramenko A.A., Kuznetsov A.V., Basok B.I., Blinov $D$. G. Investigation of stability of a laminar flow in parallelplate channel filled with a fluid saturated porous medium // Physics of flow. - 2005. - № 17. - P. 1-6.

16. Avramenko A.A., Kuznetsov A.V., Nield D.A. Instability of slip-flow in a channel occupied by a hyperporous medium // Journal of Porous Media. - 2007. - Vol. 10. - P. 435-442.

17. Nield D. A. The stability of flow in channel or duct occupied by a porous medium // J. Heat Mass Transfer.2003.- №. 46.- - P. 4351-4354. 
18. Fournier J.D., Frisch U. Remarks on the renormalization group in statistical fluid dynamics // Phys. Rev. A. - 1983. - Vol. 28, № 2. - P. 1000 - 1002.

19. Yakhot V, Orszag S.A. Renormalization group analysis of turbulence. I. Basic theory // J. Sci. Comp.1986.- 1, № 1.- P. 3-51.
20. Avramenko A.A., Kuznetsov A.V. Renormalization Group Model of Lage-Scale Turbulence in Porous Media // Transrort in Porous Media. - 2006. - № 63. - P. 175-193. 21. Avramenko A.A., Basok B.I., Dmitrenko N.P. Renormalization group analysis of turbulence. - Kyiv: Express, 2013. 298 p. 


\section{ЛИНЕЙНАЯ И НЕЛИНЕЙНАЯ НЕУСТОЙЧИ- ВОСТЬ ПОТОКА В КАНАЛЕ, ЗАПОЛНЕННОМ ПОРИСТОЙ СРЕДОЙ}

\author{
Авраменко А.А., чл.-кор. НАН Украины, \\ Дмитренко Н.П., канд. техн. наук, Ковецкая Ю.Ю. \\ Институт технической теплофизики НАН Украины. \\ 03057, г. Киев, ул. Желябова, 2а
}

Ключевые слова: ренормализационный анализ, математическая модель, пористость, неустойчивость, турбулентность

Библ. 21, рис. 2.

$\mathrm{B}$ пористой среде поток может носить ламинарный, переходной или турбулентный характер. И при этом важно знать параметры, при которых один режим течения переходит в другой. В настоящей статье исследована линейная и нелинейная гидродинамическая неустойчивость потока в канале, заполненном пористой средой. Для проведения линейного анализа неустойчивости использовалась модель Дарси-Бринкмана-Форхаймера в трехмерном приближении. Полученные данные обобщены зависимостью . Нелинейный анализ неустойчивости был проведен, используя ренормализированное выражение для коэффициента кинематической вязкости. В результате получен критерий гидродинамической неустойчивости.

Результаты проведенного исследования позволяют оптимизировать выбор характеристик (геометрических) пористой среды для реализации ее в технологических процессах.

\section{ЛИТЕРАТУРА}

1. B. V. Antohe and J. L. Lage. A general two-equation macroscopic model for incompressible flow in porous media, Int. J. Heat Mass Transf., 40, No. 13, 3013-3024 (1997).

2. D. Getachew, W. J. Minkowycz, and J. L. Lage. A modified form of the $k-\varepsilon$ model for turbulent flows of an incompressible fluid in porous media, Int. J. Heat Mass Transf., 43, No. 16, 2909-2915 (2000).

3. K. Y. Chung, K. S. Lee, and W. S. Kim. Modified macroscopic turbulence modeling for the tube with channel geometry in porous media, Numer. Heat Transf., Part A, 43, No. 6, 659-668 (2003).

4. M. J. S. de Lemos and M. H. J. Pedras. Recent mathematical models for turbulent flow in saturated rigid porous media, ASME J. Fluids Eng., 123, No. 4, 935-940 (2001).

5. M. J. S. de Lemos and M. H. J. Pedras. On the mathematical description and simulation of turbulent flow in a porous medium formed by an array of elliptic rods,
ASME J. Fluids Eng., 123, No. 4, 941-947 (2001).

6. M. J. S. de Lemos and M. H. J. Pedras. Macroscopic turbulence modeling for incompressible flow through undeformable porous media, Int. J. Heat Mass Transf., 44, No. 6, 1081-1093 (2001).

7. M. J. S. de Lemos and M. H. J. Pedras. Simulation of turbulent flow in porous media using a spatially periodic array and a low Re two-equation closure, Numer. Heat Transf., Part A, 39, No. 1, 35-59 (2001).

8. T. Masuoka, Y. Takatsu, and T. Inoue. Chaotic behavior and transition to turbulence in porous media, Microscale Thermophys. Eng., 6, No. 4, 347-357 (2002).

9. D. W. Barr. Turbulent flow through porous media, Ground Water, 39, No. 5, 646-650 (2001).

10. G. Alvarez, P. E. Bournet, and D. Flick. Twodimensional simulation of turbulent flow and heat transfer through stacked spheres, Int. J. Heat Mass Transf., 46, No. 13, 2459-2469 (2003).

11. D. Flick, A. Leslous, and G. Alvarez. Semi-empirical modeling of turbulent fluid flow and heat transfer in porous media, Int. J. Refrig., 26, No. 3, 349-359 (2003).

12. P. Vadasz. Small and moderate Prandtl number convection in a porous layer heated from below, Int. J. Energy Res., 27, No. 10, 941-960 (2003).

13. S. Hahn, J. Je, and H. Choi. Direct numerical simulation of turbulent flow with permeable walls, J. Fluid Mech., 450, 259-285 (2002).

14. H. H. Macedo, U. M. S. Costa, and M. P. Almeida. Turbulent effects on fluid flow through disordered porous media, Physica A, 299, No. 3, 371-377 (2001).

15. A. A. Avramenko, A. V. Kuznetsov, B. I. Basok, and D. G. Blinov. Investigation of stability of a laminar flow in parallel-plate channel filled with a fluid saturated porous medium. Phys. Fluids, 17, No. 9, 94-103 (2005).

16. A. A. Avramenko, A. V. Kuznetsov, and D. A. Nield. Instability of slip-flow in a channel occupied by a hyperporous medium, J. Porous Media, 10, No. 5, 435-442 (2007).

17. Nield D. A, Bejan A. Convection in porous media, 2nd ed. New York: Springer, 1999. 633 p.

18. Fournier J. D., Frisch U. Remarks on the renormalization group in statistical fluid dynamics. Phys. Rev. A. 1983. Vol. 28, № 2. P. $1000-1002$.

19. Yakhot V, Orszag S.A. Renormalization group analysis of turbulence. I. Basic theory J. Sci. Comp. 1986. Vol. 1, № 1. P. 3 - 51 .

20. A.A. Avramenko and A.V. Kuznetsov. Renormalization group model of large-scale turbulence in porous media, Transp. Porous Media, 63, No. 1, 175-193 (2006).

21. Avramenko A.A., Basok B.I., Dmitrenko N.P. Renormalization group analysis of turbulence. - Kyiv: Express, 2013. 298 p. 